\title{
Discrete gap solitons in modulated waveguide arrays
}

\author{
Andrey A. Sukhorukov and Yuri S. Kivshar \\ Nonlinear Physics Group, Research School of Physical Sciences and Engineering, \\ Australian National University, Canberra, ACT 0200, Australid
}

\begin{abstract}
We suggest a novel concept of diffraction management in waveguide arrays and predict the existence of discrete gap solitons that possess the properties of both conventional discrete and Bragg grating solitons. We demonstrate that both the soliton velocity and propagation direction can be controlled by varying the input light intensity.
\end{abstract}

(C) 2018 Optical Society of America

OCIS codes: $190.4390,190.4420$

Photonic structures with a periodic modulation of the refractive index (e.g. photonic crystals) can be used to precisely control propagation of optical pulses and beams. Wave localization is possible inside the band gaps of the linear spectrum, whereas dispersion and diffraction characteristics are strongly modified near the band edges. In particular, recent papers 123 reported on the fabrication of one-dimensional periodic arrays of identical optical waveguides where the effective diffraction coefficient can vanish or even become negative, being controlled by the input conditions and array parameters.

In comparison with homogeneous media, efficiency of nonlinear processes can be greatly enhanced in properly designed periodic structures. For waveguide arrays, where waves are primarily localized in weakly coupled waveguides, the effective diffraction can be greatly reduced, further lowering the threshold for the beam selffocusing. It was predicted that fiffractional spreading is suppressed for discrete soliton 15 that are known to possess many remarkable properties 6 . For example, unlike their continuous counterparts, discrete solitons can propagate across an array at low powers, while at high powers they become trapped by array discretenesp 6 , and this behavior is readily observed in experiment 9 . It was also demonstrated that discrete solitons can be efficiently routed through two-dimensional networks of coupled waveguides 10 .

In a number of recent studies 11.12 , it was suggested that the properties of discrete solitons can be modified

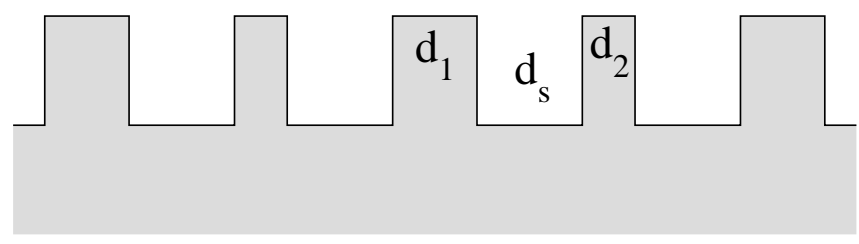

FIG. 1: Schematic of a binary array of waveguides with alternating widths $d_{1}$ and $d_{2}$ and separation $d_{s}$.

*URL: http://www.rsphysse.anu.edu.au/nonlinear by a periodic modulation of waveguides along the propagation direction. In this Letter, we suggest another, even more fundamental concept of the array engineering and consider a binary waveguide array composed of alternating "thick" and "thin" waveguides, as illustrated in Fig. 11. In such a structure, the effective refractive index experiences additional transverse modulation and, therefore, a "Rowland ghost gap" may appear in the linear spectrum13. Formation of solitons in such gaps was earlier studied in the context of superstructure fiber Bragg grating,14, where the analysis is based on the coupledmode equations. In this Letter, we demonstrate, for the first time to our knowledge, the existence of discrete gap solitons that display the properties of both conventional discrete and Bragg grating solitons and resemble nonlinear localized gap modes in diatomic lattices 15.

Propagation of waves in an array of weakly coupled single-mpde optical waveguides can be approximately described 45 by the discrete nonlinear Schrödinger equation (DNLS) for the normalized amplitude of the electric field $E_{n}$ localized at the waveguide with the index $n$,

$$
i \frac{d E_{n}}{d z}+\lambda_{n} E_{n}+\left(E_{n-1}+E_{n+1}\right)+\gamma_{n}\left|E_{n}\right|^{2} E_{n}=0,
$$

where $\lambda_{n}$ characterizes the linear propagation constant of the mode guided by the $n$-th waveguide (which depends on its width), and $\gamma_{n}$ is the effective nonlinear coefficient. For the analysis of the structure shown in Fig. 1, it is convenient to introduce the notations: $a_{n}=E_{2 n}$, $b_{n}=E_{2 n+1}, \lambda_{2 n+1} \equiv-\rho$, and $\lambda_{2 n} \equiv 0$, assuming the appropriate normalization of Eq. (1). In order to simplify the analysis, we neglect absorption and also consider the identical nonlinear coefficients, $\gamma_{n} \equiv \gamma$. However, we have verified that the main conclusions of our study remain valid if $\gamma_{n}$ are weakly modulated. With no loss of generality, we have $\rho>0$, so that $a_{n}$ and $b_{n}$ are the field amplitudes at the thick and thin waveguides, respectively. Dependence of the normalized detuning on the waveguide widths $d_{1,2}$ and the separation $d_{s}$ (see Fig. 11) can be found numerically.

Our structure fan be compared to the waveguide arrays with defects 16 , where localization at a thin waveguide $\left(d_{2}=2.5 \mu \mathrm{m}\right)$ embedded in an array of thicker waveguides $\left(d_{1}=4 \mu \mathrm{m}\right)$ with $d_{s}=5 \mu \mathrm{m}$ was recently ob- 

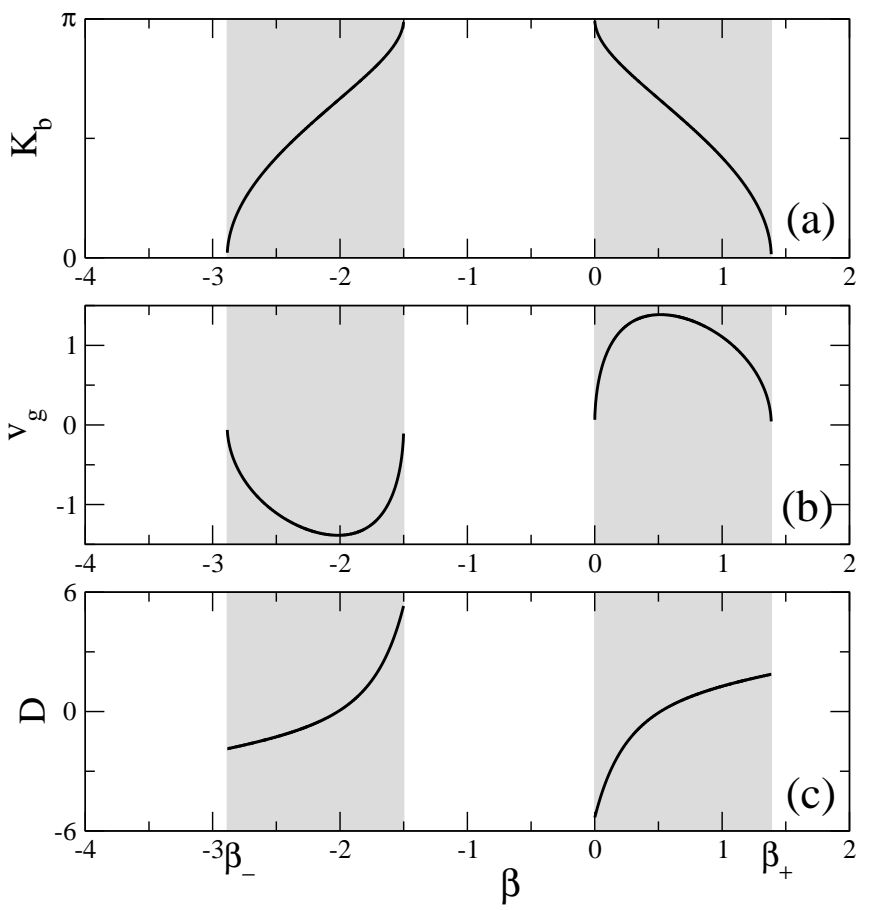

FIG. 2: Characteristic dependencies of the Bloch wave number $\left(K_{b}\right)$, group velocity $\left(v_{g}\right)$, and the effective diffraction coefficient $(D)$ on the propagation constant $\beta$. Gray shadings mark the transmission bands. Normalized detuning between the thick and thin waveguides is $\rho=1.5$.

served in experiments with AlGaAs arrays. Under such conditions, the normalized detuning between the modes of the thin and thick waveguides is about $\rho \simeq 1.5$, the value we use in numerical simulations presented belpw. Defects with other parameters can also be fabricated 17 .

First we analyze the properties of linear Bloch waves in such a periodic binary structure. The Bloch waves are characterized by the wave number $K_{b}$,

$$
a_{n}=A e^{i \beta z+i K_{b} n}, \quad b_{n}=B e^{i \beta z+i K_{b} n},
$$

where $\beta$ is the mode propagation constant. We substitute Eq. (2) into the linearized equation (11) (with $\gamma=0$ ), and obtain the linear dispersion relation that couples the propagation constant and Bloch wave number, and yields a relation between the amplitudes at the thin and thick waveguides,

$$
K_{b}=\cos ^{-1}(\eta / 2), \quad A \beta=B e^{-i K_{b} / 2} \sqrt{2-\eta},
$$

where $\eta=2-\beta(\beta+\rho)$. It follows that the transmission bands, corresponding to real $K_{b}$, appear when $\beta_{-} \leq \beta \leq$ $-\rho$ or $0 \leq \beta \leq \beta_{+}$, where $\beta_{ \pm}=-(\rho / 2) \pm \sqrt{(\rho / 2)^{2}+4}$. On the other hand an additional gap, also called the Rowland ghost gap13, appears for $-\rho<\beta<0$, and it increases for a larger difference between the widths of the neighboring waveguides. A characteristic dispersion relation and the corresponding band-gap structure are presented in Fig. 2(a).
Using the dispersion relation (3), we calculate the group velocity, $v_{g}=-2\left(\partial \beta / \partial K_{b}\right)$, and the effective diffraction coefficient, $D=-4\left(\partial^{2} \beta / \partial K_{b}^{2}\right)$, of the binary array. Characteristic dependencies of these parameters on the propagation constant $\beta$ are shown in Figs. 2(b,c). In both transmission bands, there exist regions with effective normal and anomalous diffraction separated by a zero-diffraction point.

When the medium possesses a self-focusing nonlinearity, bright discrete solitons can form provided the effective diffraction coefficient is positive. We consider excitation of such solitons by an input Gaussian beam,

$$
E_{n}(z=0)=C \exp \left[-\left(n-n_{0}\right)^{2} / \nu^{2}+i \kappa\left(n-n_{0}\right)\right],
$$

where $n_{0}$ is the position of the beam center, $\nu$ is the beam width, and $\kappa$ characterizes the inclination angle. Such an input beam can be presented as a superposition of two modulated linear eigenmodes, whose eigenvalues $\beta_{1,2}$ correspond to the Bloch wave number $K_{b}=\cos ^{-1}[\cos (2 \kappa)]$,

$$
\begin{aligned}
& E_{2 n}(0)=\left(A_{1}+A_{2}\right) \exp \left[-\left(2 n-n_{0}\right)^{2} / \nu^{2}+i K_{b} n\right], \\
& E_{2 n+1}(0)=\left(B_{1}+B_{2}\right) \exp \left[-\left(2 n+1-n_{0}\right)^{2} / \nu^{2}+i K_{b} n\right],
\end{aligned}
$$

where the amplitudes $A_{j}$ and $B_{j}(j=1,2)$ satisfy Eq. (3) at $\beta=\beta_{j}$ (we choose $\beta_{1}>\beta_{2}$, with no loss of generality). For a wide input beam $(\nu \geq 4)$, a ratio of the powers of two excited Bloch modes can be written in a simple form,

$$
p=\frac{P_{2}}{P_{1}} \simeq\left(\frac{1-\delta}{1+\delta}\right)^{2},
$$

where $\delta=\left[\sqrt{1+\rho / \beta_{1}} \cos \kappa\right] / \cos \left(K_{b} / 2\right)$. The two modes always have opposite group velocities and diffraction coefficients (see Fig. 2).

It is useful to compare the results with a homogeneous array, when $\rho=0$. In this case, within the validity of the DNLS model, there exists only one parameter domain where diffraction is positive, and discrete solitons can exist, for self-focusing medial, (t). On the other hand, in a modulated array diffraction is modified close to the Rowland ghost gap, and there appear two regions with positive diffraction, where solitons can exist. Thus, the binary array structure allows the band gap engineering in a broad parameter region.

Let as first discuss the excitation of discrete solitons by a beam incident at a normal angle, so that $\kappa=K_{b}=0$, and $\beta_{1,2}=\beta_{+,-}$. In this case, $p<1$, i.e. the first $(j=1)$ mode is always dominant (for example, $p \simeq 0.03$ for $\rho=1.5)$. As is demonstrated in Fig. 2, at $\beta=\beta_{+}$ the effective diffraction coefficient is positive and, therefore, discrete solitons can be formed in a self-focusing medium. Properties of such solitons are similar to those in homogeneous arrays.

On the other hand, discrete gap solitons may appear due to localization withing the linear gap (at $-\rho<\beta<$ 0 ), which appears due to a peridic modulation of waveguide widths as discussed above. In order to excite stationary gap solitons in a self-focusing medium, one might 

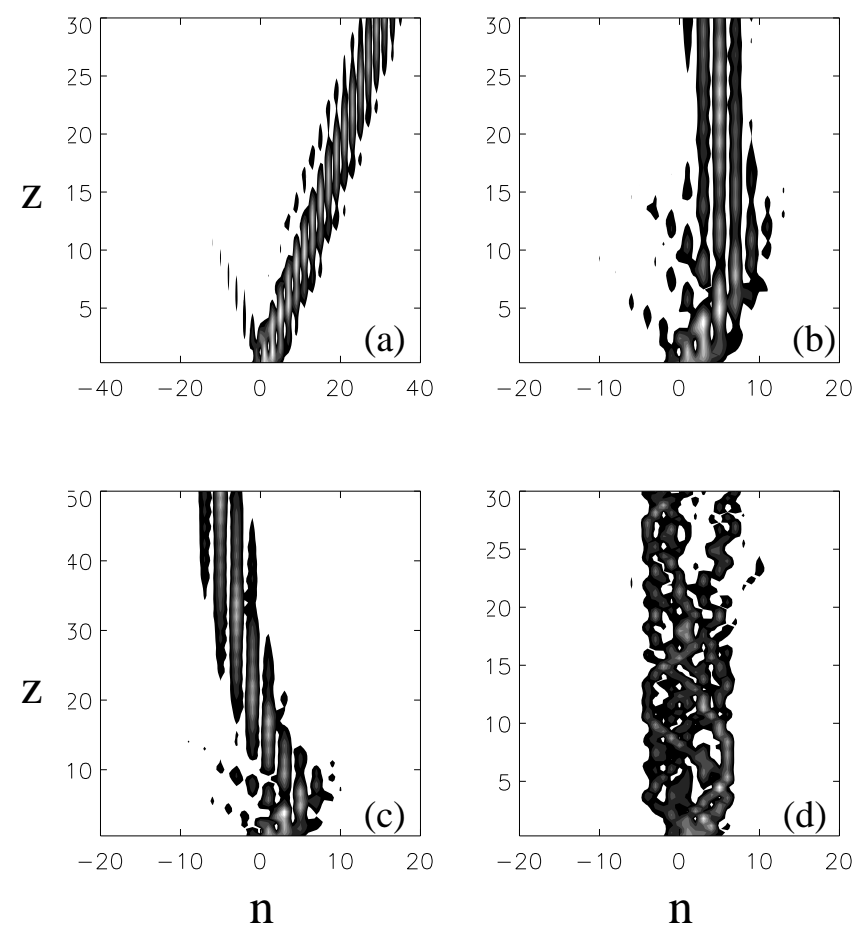

FIG. 3: Excitation of discrete gap solitons in a self-focusing medium $(\gamma=+1)$ by input beams with $\kappa=0.6 \pi, n_{0}=1, \nu=$ 4 , and normalized peak intensities (a) $|C|^{2}=0.3$, (b) 0.75 , (c) 0.9, (d) 5 . The parameters correspond to Fig. 2 .

attempt to use an input beam with $\kappa=\pi / 2$, so that $K_{b}=\pi, \beta_{1}=0, \beta_{2}=-\rho$. In this case, two Bloch modes have equal powers $(p=1)$, however one of them experiences self-focusing and another one - self-defocusing, and in this situation an efficient generation of gap solitons is not possible.

The optimum conditions for generating discrete gap solitons can be realized when $\pi / 2<\kappa<\pi$, and $D\left(\beta_{2}\right)>$ 0 . Indeed, under such conditions $p>1$, i.e. the second mode, which experiences self-focusing, is dominant. Moreover, the Bloch wave envelopes have opposite group velocities, so that they move apart in the opposite directions. In Fig. 3(a), we show that two beams are indeed generated at the input. The beam which moves to the right is localized at odd (i.e. thin) waveguides, and it transforms into a gap soliton. On the contrary, the other beam moves to the left, and it experiences self-defocusing and broadens.

Stationary gap solitons can be generated at higher input intensities. Under such conditions, two Bloch modes are initially trapped together, resulting in a periodic beating that can strongly affect the soliton formation process. If the emerging soliton has a small velocity, it is not able to overcome the Peierls-Nabarro potential of the periodic structure, and it becomes trapped $\mathrm{B}$. In this case, the gap soliton can remain in the center, as is shown in Fig. 3(b). However, if the soliton acquires a higher velocity, it can still move across the array, as is shown in Fig. 3(c). Somewhat surprisingly, in the latter case the soliton moves to the left, i.e. in the direction opposite to the propagation direction of the input beam. If the intensity is increased even further, then the gap solitons become oscillatory unstable, similar to the solitons in fiber Bragg gratings 18 [see Fig. 3(d)].

In conclusion, we have studied the diffraction properties and nonlinear wave propagation in binary waveguide arrays with alternating waveguide widths. We have predicted the existence of discrete gap solitons and demonstrated their intriguing dynamics controlled by varying the input intensity.

The authors thank J. S. Aitchison, R. Morandotti, and Y. Silberberg for useful discussions. This work was supported by the Australian Research Council.

\section{References}

[1] H. S. Eisenberg, Y. Silberberg, R. Morandotti, and J. S. Aitchison, "Diffraction management," Phys. Rev. Lett. 85, 1863-1866 (2000).

[2] R. Morandotti, H. S. Eisenberg, Y. Silberberg, M. Sorel, and J. S. Aitchison, "Self-focusing and defocusing in waveguide arrays," Phys. Rev. Lett. 86, 3296-3299 (2001).

[3] T. Pertsch, T. Zentgraf, U. Peschel, A. Brauer, and F. Lederer, "Anomalous refraction and diffraction in discrete optical systems," Phys. Rev. Lett. 88, 093901-4 (2002).

[4] D. N. Christodoulides and R. I. Joseph, "Discrete selffocusing in nonlinear arrays of coupled wave-guides," Opt. Lett. 13, 794-796 (1988).

[5] Yu. S. Kivshar, "Self-localization in arrays of defocusing wave-guides," Opt. Lett. 18, 1147-1149 (1993).

[6] F. Lederer, S. Darmanyan, and A. Kobyakov, "Discrete solitons," in Spatial Optical Solitons, Vol. 82 of Springer series in optical sciences, S. Trillo and W. E. Torruellas, eds., (Springer-Verlag, New York, 2001), pp. 269-292.

[7] C. Claude, Yu. S. Kivshar, O. Kluth, and K. H. Spatschek, "Moving localized modes in nonlinear lattices," Phys. Rev. B 47, 14228-14232 (1993).

[8] A. B. Aceves, C. De Angelis, T. Peschel, R. Muschall, F. Lederer, S. Trillo, and S. Wabnitz, "Discrete selftrapping, soliton interactions, and beam steering in nonlinear waveguide arrays," Phys. Rev. E 53, 1172-1189 (1996).

[9] R. Morandotti, U. Peschel, J. S. Aitchison, H. S. Eisenberg, and Y. Silberberg, "Dynamics of discrete solitons in optical waveguide arrays," Phys. Rev. Lett. 83, 27262729 (1999).

[10] D. N. Christodoulides and E. D. Eugenieva, "Blocking and routing discrete solitons in two-dimensional networks of nonlinear waveguide arrays," Phys. Rev. Lett. 87, 233901-4 (2001).

[11] M. J. Ablowitz and Z. H. Musslimani, "Discrete diffraction managed spatial solitons," Phys. Rev. Lett. 87, 254102-4 (2001).

[12] U. Peschel and F. Lederer, "Oscillation and decay of discrete solitons in modulated waveguide arrays," J. Opt. Soc. Am. B 19, 544-549 (2002). 
[13] P. St. J. Russell, "Bragg resonance of light in optical superlattices," Phys. Rev. Lett. 56, 596-599 (1986).

[14] N. G. R. Broderick, C. M. de Sterke, and B. J. Eggleton, "Soliton solutions in Rowland ghost gaps," Phys. Rev. E 52, R5788-R5791 (1995).

[15] Yu. S. Kivshar and N. Flytzanis, "Gap solitons in diatomic lattices," Phys. Rev. A 46, 7972-7978 (1992).

[16] U. Peschel, R. Morandotti, J. S. Aitchison, H. S. Eisenberg, and Y. Silberberg, "Nonlinearly induced escape from a defect state in waveguide arrays," Appl. Phys. Lett. 75, 1348-1350 (1999).
[17] R. Morandotti, H. S. Eisenberg, D. Mandelik, Y. Silberberg, D. Modotto, M. Sorel, and J. S. Aitchison, "Interactions of Discrete Solitons with Defects and Interfaces," In Quantum Electronics and Laser Science Conference, OSA Technical Digest, Postconference ed. OSA Trends in Optics and Photonics (TOPS) 74, 239 (Optical Society of America, Washington DC, 2002).

[18] I. V. Barashenkov, D. E. Pelinovsky, and E. V. Zemlyanaya, "Vibrations and oscillatory instabilities of gap solitons," Phys. Rev. Lett. 80, 5117-5120 (1998). 